\title{
Design of Web-Based Experiments on Acceleration and Speed Transducers
}

\author{
Ashish Mani, ${ }^{1}$ R. S. S. Prasanth, ${ }^{2}$ and C. Patvardhan ${ }^{3}$ \\ ${ }^{1}$ USIC, Dayalbagh Educational Institute, Dayalbagh, Agra 10, India \\ ${ }^{2}$ Technical College, Dayalbagh Educational Institute, Dayalbagh, Agra 10, India \\ ${ }^{3}$ Faculty of Engineering, Dayalbagh Educational Institute, Dayalbagh, Agra 10, India
}

Correspondence should be addressed to Ashish Mani, ashish.mani@rediffmail.com

Received 26 August 2010; Accepted 10 January 2011

Academic Editor: Phillip J. Belfiore

Copyright (c) 2011 Ashish Mani et al. This is an open access article distributed under the Creative Commons Attribution License, which permits unrestricted use, distribution, and reproduction in any medium, provided the original work is properly cited.

\begin{abstract}
Remote laboratories are becoming increasingly popular in academic arena due to availability of web-based technologies and infrastructure. Remote Labs are equipped with real instruments, where experiments are performed by controlling the instruments and observing the real data from a distant location through computer networks. They provide for student centric laboratory experience as students can perform experiments at their own convenience and requirements. Further, Remote Labs reduce management of work involved in running conventional laboratories. This paper describes design and implementation of such an experimental setup for performing characterization experiments on Transducers in general and Acceleration and Speed Transducers in particular from a remote location. The experiment can be made accessible to anyone over a TCP/IP network with a standard Web Browser, relevant plugin, and permission. This paper demonstrates that traditional experiments on sensors and transducers can be successfully made available online to both on-campus and off-campus students at their own schedule and convenience.
\end{abstract}

\section{Introduction}

The unprecedented advancements in Information and Communication Technologies (ICTs), availability of quality infrastructure, and inexpensive services have led to exponential expansion of applications of ICTs in almost all fields of endeavor. ICTs have brought about significant changes in lifestyle of individuals by providing easy connectivity and access to information. The world of education has also been greatly affected by advancements in ICTs. It has enabled variety of new avenues and methodologies for enhancing the experience of learning and teaching in both types of programs for imparting education, namely, Traditional Classroom and Distance Education (DE) Program. Therefore, ICTs have immense potential to make science and engineering education more affordable for larger pool of students.

It is well known that relevant laboratory experience can significantly improve students' learning and interest level in course material [1-3]. This is particularly true for courses like Science and Engineering. The students of science and engineering need laboratories for measurement, data collection, analysis, and experience of handling equipment, and other physical devices [1]. The laboratory experience is essential for developing better understanding, problem solving skills, and skills of minute observation in students. However, the effective integration of relevant experiments into courses remains the most challenging aspect of any program due to constraints of time and space and limited resources available for provision of laboratory infrastructure.

A cost-effective and technologically feasible solution to this problem is to use Information and Communication Technologies for providing Laboratory experience. Student centric Remote Labs can make learning more easy and effective. Remote Labs are equipped with real instruments, where experiments can be performed by controlling the instruments and observing the real data from a distant location through the network. Students can conduct experiments from any computer on the network at their own schedule. It can provide for efficient sharing of expensive 
laboratory resources. Remote Labs can significantly reduce the managerial work involved in conventional labs, which includes scheduling of classes, staff, equipment, lab space, training, and safety provisions [4].

Many universities have developed Internet-based remote laboratories [4-19]. Most of the efforts on implementation of Remote Labs have been related to study of control systems [4-12] and to a certain extent in study of Electronics Engineering [13-19]. However, in this paper, we are concerned with experiments related to study of Acceleration and Speed Transducers.

There are different approaches of developing Remote Laboratories using LabVIEW as illustrated in $[12,20]$ as well as other proprietary platforms $[4,7,15]$.

LabVIEW is one of the well-known packages used for development of Remote Labs. Most of the work reported so far uses Internet Toolkit, DataSocket, and so forth for developing Remote Labs, which require clients to have software developed in LabVIEW [10]; that is, Fat Clients are required. Fat Client implies that student's computer is required to have powerful hardware for running the presentation as well as the processing modules of the Experiments in the Remote Lab software, while the Thin Server would only host the Instruments and manage communication. In contrast, this paper focuses on using the Thin Client technology in development of the Remote Lab, which needs a standard web browser with relevant plugins running on any type of minimal hardware platform without any specific requirements. This simplifies the client side, so that students can concentrate on experiments rather than on learning the client interface. Further, the logistic involved is also simplified as the student is no longer required to obtain and install the experiment-specific software. It is also less expensive.

The work reported in this paper is mainly concerned with some issues in setting up of a remote experimental setup for characterization of Acceleration and Speed Transducers. There are two modes in which experiments can be performed, namely, Live Mode and File Mode. In Live Mode, the student gains control of the experimental hardware setup; therefore, only one student can perform the experiments at a time, but others can view it. However, in File Mode, many students can perform experiments simultaneously. File Mode is different and better than simulations as real experimental data, which were stored in a file from previous similar experimental runs, are provided.

If students perform experiments in File Mode, they gain understanding of how to do the experiments. Further, if they either perform experiments or view experiments being performed in Live Mode, then they gain complete understanding and feeling of the actual experiments. The inclusion of the two modes reduces waiting time of students for performing live experiments and increases effective utilization of experimental setups as viewing also becomes a significant contributor to learning process [21]. The experiments on characterization of accelerometer and tachogenerator are currently available in both Live and File Modes.

The rest of the paper is organized as follows. Section 2 describes overall system architecture. The hardware part is discussed in Section 3. In Section 4, software developed is described. Results, conclusions and future work are discussed in Sections 5 and 6, respectively.

\section{System Architecture}

The generic system architecture of the Remote Lab is shown in Figure 1. The Lab Server is interfaced with experimental setups through Data Acquisition (DAQ) Cards. It hosts software programs, web servers, and web pages of experiments and also provides connectivity with the Institute's LAN. The Client computers connected on the Institute's LAN can access experimental setup available in Remote Lab. Universities/Institutions have Internet connectivity, which is generally provided by Computer Center through Proxy and Firewall Servers acting as Internet Gateway. Thus, the Remote Lab facility can be extended outside the University Campus by Internet through Computer Center.

The proposed architecture is modular and facilitates easy integration of traditional experiments on characterization of sensors and transducers. This is illustrated by implementing the experiments on characterization of acceleration and speed transducers in this architecture. The objective of characterization experiment on Acceleration transducer is to determine the output voltage of accelerometer (along with its signal conditioner) with changes in acceleration. The objective of the Speed Sensor's characterization experiment is to determine the output voltage of tachogenerator (along with its signal conditioner) with changes in speed. Therefore, the experimental setup should be able to generate controlled variable acceleration and angular velocity. The generation of controlled angular velocity is achieved by using a DC motor, which is powered by a Programmable Power Supply (PPS). A compound lever (connecting rod-crank) mechanism driven by the DC motor is used for generating acceleration. The accelerometer, whose characteristic is to be determined, is mounted on the slider of the compound lever. The tachogenerator, whose characteristic is to be determined, is mounted on the shaft of the DC motor. Further, an optical system is used as standard measure of angular velocity for characterizing the sensors [22]. The speed of DC motor is varied by changing the output of Programmable Power Supply, which is interfaced to the Lab Server through Analog Output channel of DAQ card. The sensors are also interfaced through their respective signal conditioners to the Lab Server by using DAQ card's analog input channels so that the output voltage waveform of the sensors can be measured. The readings of speed (from which acceleration is computed), acceleration, and corresponding output voltage of accelerometer and tachogenerator are processed and recorded to plot the characteristic curves. Further, the values of speed, acceleration, and corresponding output voltages can be stored for future reference.

A software-based controller written in LabVIEW is used for controlling the speed of the motor during the experiment. The Graphical User Interface (GUI) of the experiments is developed using LabVIEW as it provides rich repository of customizable objects. The web pages are developed from 


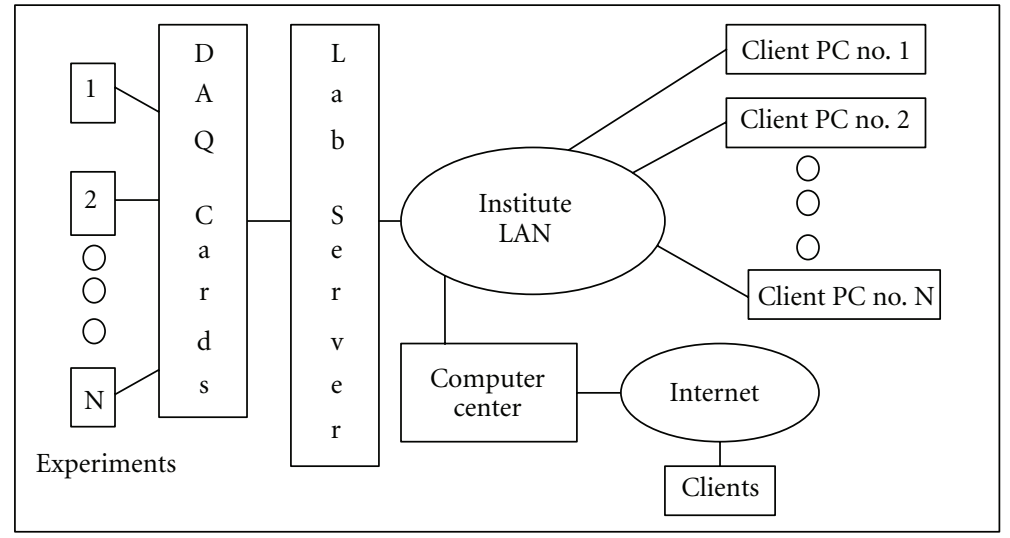

FIGURE 1: System architecture of remote lab.

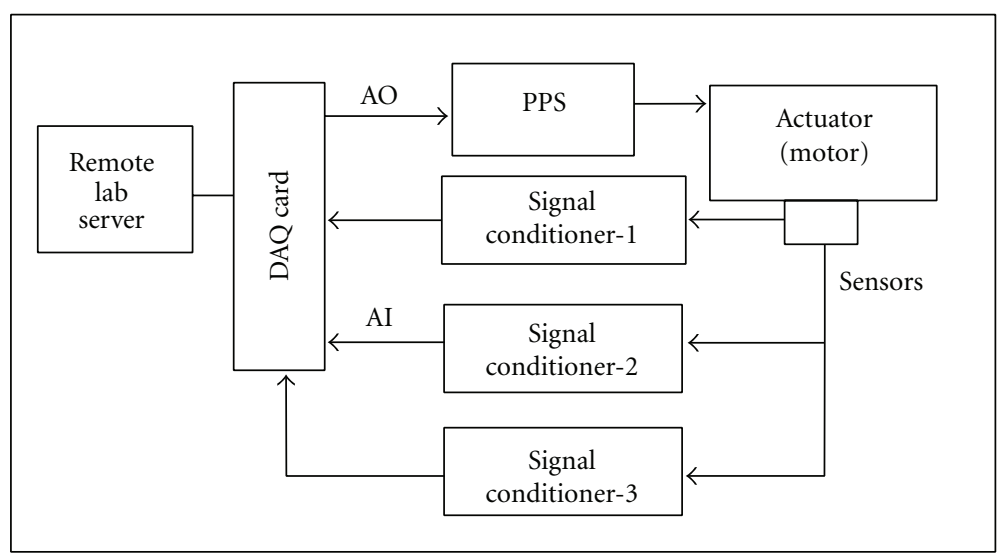

Figure 2: Hardware block diagram of remote experimental setup.

Virtual Instruments (VIs) programs using Web Publishing tools in LabVIEW. LabVIEW Web Server is used for hosting web pages. The G Web server is used for handling CGI requests. The server programs are also developed using LabVIEW. This experimental setup can be controlled from any remote PC connected on LAN/Internet. The Industry Standard TCP/IP protocol is used for communication between remote PC and Lab Server.

The experiments can be performed in the two modes as discussed in the previous section. In case of Live Mode, the experimental hardware is available for controlling purposes to only one student at a time, but others can view it. The hardware is illustrated in Section 3. However, In case of File Mode, the traditional experimental setup's hardware is replaced with a file, which has the real data stored from previous Live Mode experimental runs. Therefore, the traditional experimental setup's hardware is not required, so many students can perform experiments simultaneously. File Mode is illustrated in Section 4.

\section{Hardware}

The hardware block diagram of the web-based experimental setup in Remote Lab is shown in Figure 2. The hardware used in implementing the web-based experimental setup are Accelerometer, Tachogenerator, Optical Transducer, Signal Conditioners, DC Motor, Compound Lever (Connecting Rod-Crank) mechanism, Programmable DC Power Supply, DAQ card, Lab Server, Network, and Remote Computers.

3.1. Accelerometer and Its Signal Conditioner. The accelerometer is an electromechanical transducer, which provides an electrical output proportional to the vibratory acceleration it undergoes. The accelerometer used in this experimental setup is a Preamplified Piezoelectric accelerometer, which is normally used for automotive applications like triggering of air bags in cars. It can measure acceleration $\pm 1000 \mathrm{~g}$, where $1 \mathrm{~g}=9.81 \mathrm{~ms}^{-2}$ with sensitivity of $4 \mathrm{mV} / \mathrm{g}$ nominal. The resonance frequency is $10 \mathrm{kHz}$, output voltage is $4 \mathrm{~V} \pm 2.5 \mathrm{~V}$, and output impedance is $600 \Omega$ [22].

A signal conditioner is required to transform the high output impedance of the accelerometer into lower output impedance. It also amplifies and filters the voltage signal generated by the accelerometer. It includes charge amplifier to amplify the signal for obtaining a standard output of 0 to $8 \mathrm{~V}$ peak to peak (pp) and a filter for eliminating highfrequency signals of greater than $1 \mathrm{KHz}$. It also eliminates direct current offset from the output of preamplifier included 
in the accelerometer. It detects vibrations in both positive and negative ranges and also provides buffer between the transducer and the DAQ card. It is calibrated so that an acceleration of $80 \mathrm{~g}$ corresponds to $8 \mathrm{~V}$ peak to peak.

The mounting of accelerometer is important as it directly affects reliability of readings at high acceleration. The accelerometer used in this experimental setup is mounted horizontally on the Compound Lever and is firmly fixed with a small steel screw [22].

3.2. Tachogenerator and Its Signal Conditioner. The devices, which convert the speed into electrical quantity, are called speed transducers like tachogenerator. It consists of a stator, which has a permanent magnet and a rotor with armature coil wounded on it. The armature coil is rotated in the presence of magnetic field to develop potential difference, which is proportional to its angular velocity. The output voltage is obtained between two brushes sliding on a commutator. The tachogenerator used in this experimental setup has linearity of $0.2 \%$, ripple is $2.0 \%$ at $500 \mathrm{rpm}$, ripple frequency is 21 cycles per revolution, and operating temperature range is $0-155^{\circ} \mathrm{C}$. The output voltage gradient is $14.5 \mathrm{mV} / \mathrm{rpm}$, nominal armature resistance and inductance are $110 \mathrm{ohm}$ and $8 \mathrm{mH}$, respectively, and recommended load resistance is $10 \mathrm{~K}$ [22].

A signal conditioner is required to reduce noise and improve linearity of the output voltage signal and consists of potential divider, low pass filter, and amplifier. It produces standard output voltage corresponding to $\pm 4000 \mathrm{rpm}= \pm 8 \mathrm{~V}$ [22].

3.3. DC Motor, Compound Lever, and Optical System. It is necessary to generate a variable standard acceleration and speed for a proper study of the acceleration and speed transducers. The acceleration and speed transducer module by $\mathrm{M} / \mathrm{s}$ Elettronica Veneta has been used, which consists of DC motor, Compound Lever, and Optical System. The DC motor and compound lever are used for generating variable acceleration. The optical system is used for measuring angular velocity. The DC motor has a maximum speed of $4000 \mathrm{rpm}$ with a rated supply voltage of $27.5 \mathrm{~V}$. Intermediate speeds can be generated by varying the supply voltage from 0 to $27.5 \mathrm{~V}$. However, the heavy load of inversion movements on motor due to its coupling with compound lever limits its maximum speed to $800 \mathrm{rpm}$. Further, increasing the terminal voltage of the motor, increases the current and exceeds its maximum limit of $2 \mathrm{~A}$.

Compound Lever mechanism consisting of connecting rod and crank is used for generating the required acceleration. The relation between the angular speed of the motor and the acceleration of the compound lever has been derived in [22], and the equation is as follows:

$$
g=2 n^{2} * \frac{K}{9.81}
$$

where $g$ is acceleration, $n$ is angular speed in $\mathrm{rpm}$, and $K=$ $603 * 10^{-6}$.

Optical System is required for measurement of the angular velocity of the motor, which is an optical encoder mounted on the motor's shaft. It generates pulses corresponding to the angular velocity of the motor.

3.4. Programmable DC Power Supply. The Programmable DC Power Supply is used for controlling the speed of the motor. It receives input from the DAQ card's Analog Output channel, which can vary from 0 to $5 \mathrm{~V}$ to control the corresponding output voltage from 0 to $30 \mathrm{~V}$. The maximum current that can be drawn from the power supply is limited to $2 \mathrm{~A}[23]$.

3.5. Lab Server and DAQ Card. The lab server consists of a PC with Pentium IV, $2.8 \mathrm{GHz}$ processor, $1 \mathrm{~GB}$ RAM, Network Interface Card, and National Instruments DAQ Board PCI6070E, which has been used for interfacing the experimental setup along with SC-2075 Bread Board Connector Block. The server is connected to DEI-NET, Dayalbagh Educational Institute's LAN. The DAQ Card PCI 6070E has 8 Differential Input analog channels which can accept $\pm 10 \mathrm{~V}$ input at maximum sampling rate of $1 \mathrm{MS} / \mathrm{s}, 2$ Analog output channel which can give $\pm 10 \mathrm{~V}$ output and is suitable for this application.

3.6. Network and Remote Computers. DEI-NET has backbone of Fiber optic cable, which runs to all Departments' and Centres' local switches. The end PCs are connected through CAT 5e cables. In most of the Labs, Wireless networking is also available. Different configurations of Pentium-based computers including Laptops with Windows Operating System and Internet Explorer have been used for performing experiments in both Live and File Modes.

\section{Software}

The software block diagram of the experimental setup is shown in Figure 3. The software for the characterization experiments are developed using LabVIEW. The software consists of Graphical User Interfaces (GUIs), Functional Programs, VI, Web and G Web Servers, and Remote Panel Connection Manager for administration of clients.

4.1. Graphical User Interface. An intuitive GUI with steep and short learning curve is essential for any web-based experimental facility as it would not only facilitate in performing experiments but would also encourage the students to perform experiments without any supervision and traditional help. The programs developed in LabVIEW are known as Virtual Instrument (VI), which have two main parts, namely, front panel and block diagram. The front panel can be used to develop intuitive GUI as LabVIEW provides rich inbuilt and customizable objects.

There are three levels of GUI, namely, Authentication GUI, Experiment Selection and Authorization GUI, and Experiment-specific GUIs. The Authentication GUI is used for logging in the Remote Lab. The Experiment Selection and Authorization GUI is used for gaining control of the experiment to be performed in Live or File Mode. The Experimentspecific GUIs are used for performing the experiments. 


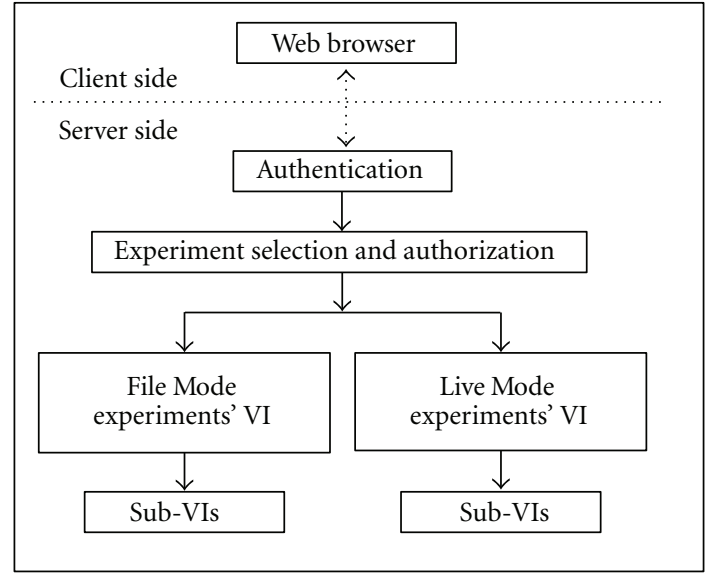

FIGURE 3: Software block diagram of web-based experimental setup.

The Experiment-specific GUI for characterization of tachogenerator is shown in Figure 4. It consists of indicators, which are showing the output voltage measured from tachogenerator circuit (including signal conditioner) and the speed as measured by Optical Transducer (calibrated circuit with its signal conditioner). There is an option for plotting the characteristic curve, while the experiment is being conducted, in the graphical indicator. It has option for saving the measurements in a spreadsheet file, whose path is shown in the indicator.

4.2. Functional Program Description. Lab Server program is used for authenticating and authorizing the students for performing experiments in Live or File Mode. If a request for performing experiment in Live Mode is made and the experimental setup is free, then it is assigned for a duration of 10 minutes. If the experimental setup is not available for controlling purpose, the request is put on hold and would be catered as soon as the experimental setup could be made available for controlling purpose. Optionally, the student can view an ongoing Live experiment. Alternatively, the student can perform the experiment in File Mode as these can be performed concurrently.

After getting authorization to perform experiment in Live Mode, the Experiment VI is launched. The student is expected to set the speed in the Set-Speed control box at which the transducer's output is to be recorded. The voltages are read from two analog input channels AI-0 and AI-1 at every $200 \mathrm{~ms}$ interval, which are connected to the acceleration and speed sensors' signal conditioners' outputs, respectively. The output voltage waveform of Optical System's signal conditioner's output is collected for ten seconds at a sampling frequency of $8 \mathrm{KS} / \mathrm{s}$. It is processed to determine the speed of the motor, which is converted to acceleration by (1).

The readings of computed acceleration from optical system's output and output voltage from acceleration transducer $\left(V_{\text {in }}\right)$ are displayed at the set speed. The acceleration transducer's characteristic curve is a plot of the set of values of $\mathrm{g} \mathrm{V} / \mathrm{s} V_{\text {in }}$. The speed of the motor can be changed from the GUI by using the Set-Speed control box. The controller takes the input from the Set-Speed control and the measured speed to determine the control action. It controls motor speed through analog output of DAQ card, which in turn controls the output of Programmable DC Power supply. The settings of controller are preset to reduce the complexity of the experiment.

The graphical indicator can show the trail on the graph by plotting all the previous values of $g$ or $v$ (depending on the experiment) and $V_{\text {in }}$ in every iteration, if the option Show Trail is selected. Alternatively, it can display only the point corresponding to the present value of $g$ or $v$ and $V_{\text {in }}$ by only plotting the present values in every iteration, if the option Hide Trail is selected.

The readings of $g$ or $v$ and $V_{\text {in }}$ can be saved in a spreadsheet file if the option Save is selected. The path of spreadsheet file is displayed in an indicator in GUIs. The file name is asked from the student, and file by the name is opened with a reference number in an FTP accessible directory. If the user selects to store the data in the file, the reference number is used for further writing to the file. When VI finally terminates execution, it closes the file. This file can be obtained by a distant user through FTP services.

4.3. LabVIEW Internet Toolkit, $G$ and Web Server, and RP Connection Manager. LabVIEW Web Server is used for hosting the Web pages. The Web pages are mostly created using Web Publishing Tool, which creates an HTML document of front panel. The G Server is used for handling CGI request. The CGI protocol is implemented using Internet Toolkit. The CGI protocol is used for dynamically creating Web pages and also for launching the VIs. The Remote Panel Connection Manager is used for administering the VI for viewing and control by remote browser clients.

\section{Results}

The prototype experimental setup has been made available to a group of students from the Faculty of Engineering, pursuing the course on Instrumentation. They have been able to perform the experiments both in Live and File Modes successfully. The responses received from the students were very encouraging; however, they were more inquisitive and keen on learning about the implementation of the Remote experimental setup. The snapshot of the experiment performed in Live Mode on the Acceleration Transducer's characterization is shown in Figure 5. The experimental data, which were saved during the experiment in file, can be used to plot the characterization curve offline using MS-Excel program. Thus, it would be helpful in preparation of reports.

\section{Conclusions and Future Work}

Laboratory experience is an important requirement of Engineering and Science Education. However, there are known problems associated with provision of lab experience in traditional environment due to paucity of space, time, and resources. A cost-effective and technologically feasible 


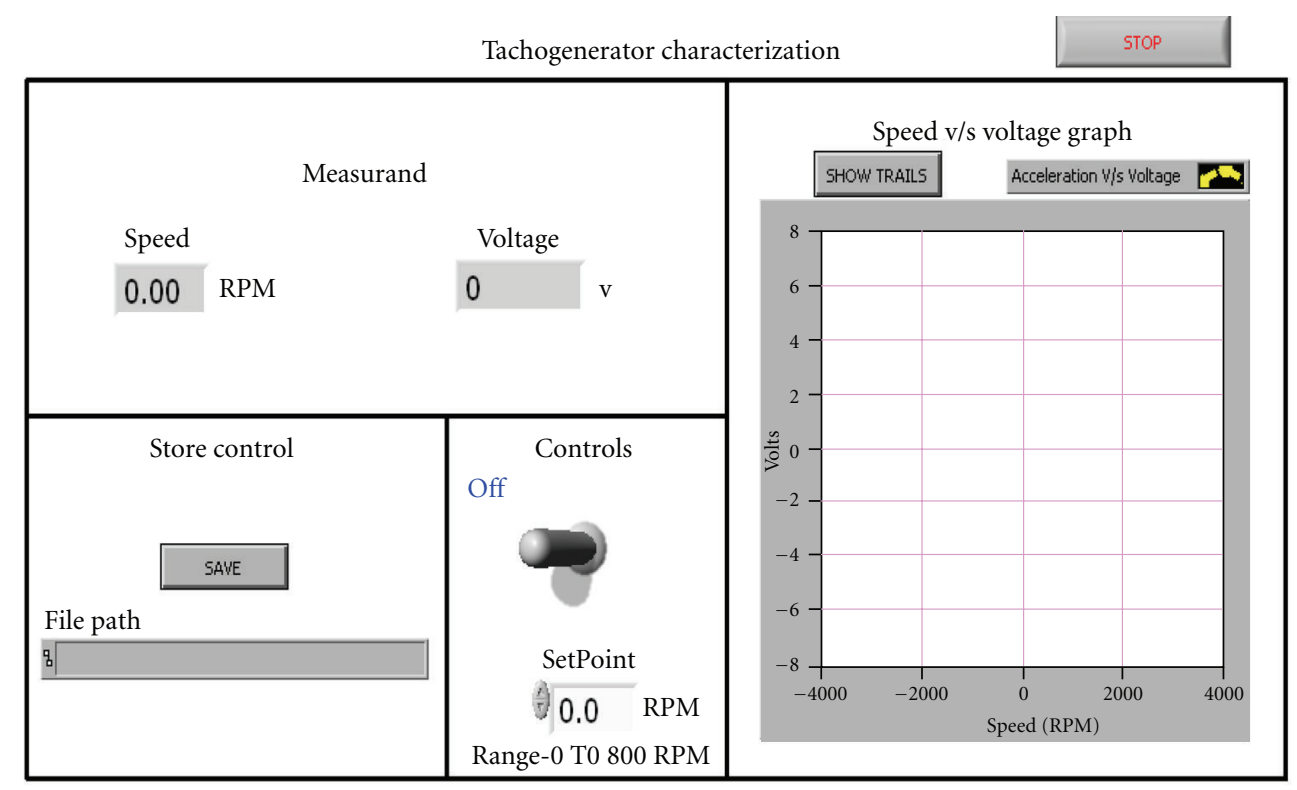

FIGURE 4: GUI for tachogenerator characterization experiment.

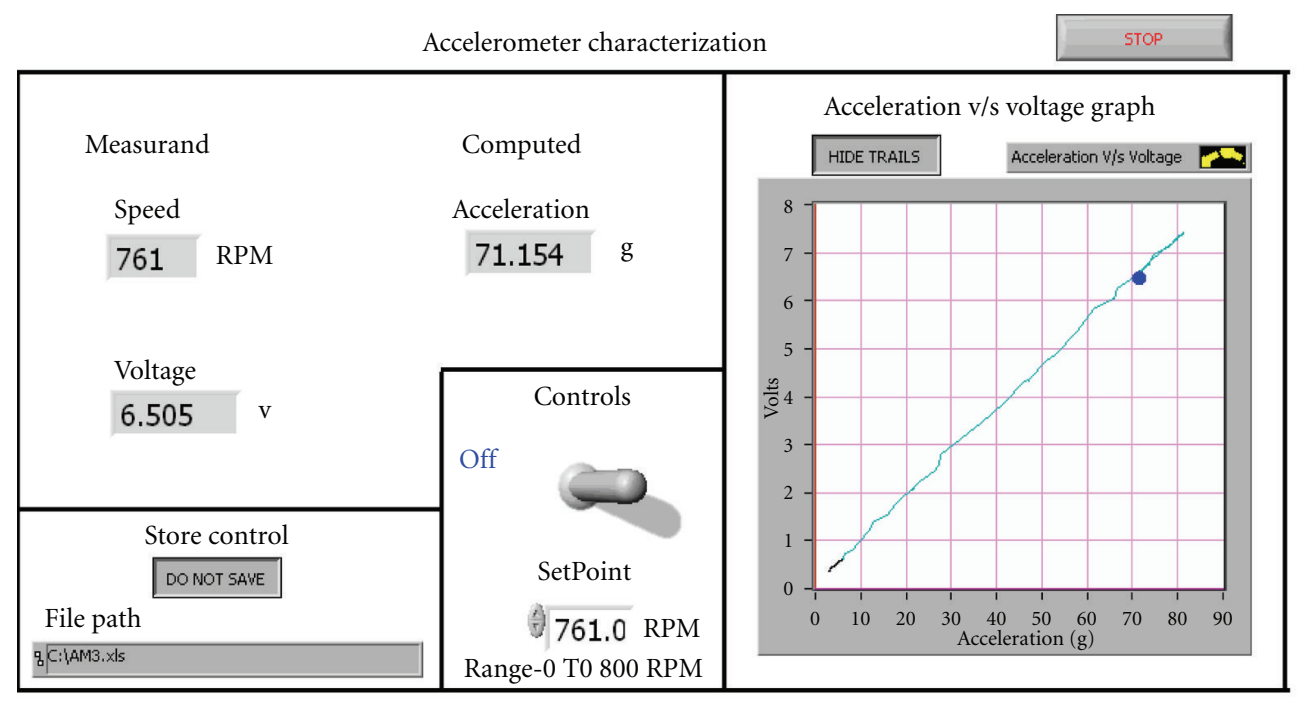

FIGURE 5: Snapshot of accelerometer characterization experiment in Live Mode.

solution to this problem is to use web-based Remote Laboratories for addressing limitations associated with traditional labs. A web-based experimental setup has been successfully developed for performing experiments on Characterization of Acceleration and Speed sensors. It demonstrates that traditional experiments, especially in the field of sensors and transducers can be made available in Remote Labs.

It provides students with flexibility to perform experiments at their own schedule and convenience. There are two modes available for performing experiments, namely, Live and File Modes.

The architecture and framework designed in this paper can be employed for making the experiments on other types of sensors and transducers available online as it is highly modular. Remote Lab has been divided into two modules, namely, Hardware and Software. The hardware module has been further subdivided into sensor modules, signal conditioner modules, Data Acquisition Cards, and the Computer hosting Remote Lab server. The software module has been further subdivided into LabVIEW Web and G Servers and experiment-specific programs. The traditional experiments can be easily integrated by determining the procedure, various excitations and responses in an experiment. The procedure of an experiment is implemented through VI programs developed in LabVIEW. The excitations are controlled through analog and/or digital outputs from the DAQ cards. The responses are measured through analog and/or digital inputs of DAQ Cards. Thus, the traditional 
experiments can be made available online by engineering the hardware modules for the experimental setup, sensors, signal conditioners, and Data Acquisition Cards, which are available as off-the-shelf components. The software can be easily developed for specific experiments by using LabVIEW.

Future work would focus on including experiments on different types of sensors and transducers like characterization of pressure, flow, and temperature sensors in the webbased Remote Lab.

\section{Acknowledgments}

The authors are extremely grateful to Most Revered Chairman Advisory Committee on Education, Dayalbagh, for continued guidance and support in their every endeavor. They are thankful to anonymous reviewers for giving valuable feedback towards improving the paper.

\section{References}

[1] L. D. Feisel and A. J. Rosa, "The role of the laboratory in undergraduate engineering education," Journal of Engineering Education, vol. 94, no. 1, pp. 121-130, 2005.

[2] N. S. Edward, "The role of laboratory work in engineering education: student and staff perceptions," International Journal of Electrical Engineering Education, vol. 39, no. 1, pp. 1119, 2002.

[3] R. Mitchell, J. Fischer, and J. A. del Alamo, "A survey study of the impact of a MIT microelectronics online laboratory on student learning," in Proceedings of the International Conference on Engineering Education (ICEE '06), San Juan, Puerto Rico, USA, 2006.

[4] G. Viedma, I. J. Dancy, and K. H. Lundberg, "A web-based linear-systems iLab," in Proceedings of the American Control Conference (ACC '05), pp. 5139-5144, June 2005.

[5] C. C. Ko, B. M. Chen, J. Chen, J. Zhang, and K. C. Tan, "A web-based laboratory on control of a two-degrees-of-freedom helicopter," International Journal of Engineering Education, vol. 21, no. 6, pp. 1017-1030, 2005.

[6] M. Casini, D. Prattichizzo, and A. Vicino, "The automatic control telelab: a user-friendly interface for distance learning," IEEE Transactions on Education, vol. 46, no. 2, pp. 252-257, 2003.

[7] F. Naghdy, P. Vial, and N. Taylor, "Embedded internet laboratory," International Journal of Engineering Education, vol. 19, no. 3, pp. 427-432, 2003.

[8] J. Henry and C. Knight, "Modern engineering laboratories at a distance," International Journal of Engineering Education, vol. 19, no. 3, pp. 403-408, 2003.

[9] D. Gillet, F. Geoffroy, K. Zeramdini, A. V. Nguyen, Y. Rekik, and Y. Piguet, "The cockpit: an effective metaphor for Web-based experimentation in engineering education," International Journal of Engineering Education, vol. 19, no. 3, pp. 389-397, 2003.

[10] E. K. Rézaei and S. R. Kolla, "Internet-based ON/OFF controller using labVIEW," in Proceedings of the ASEE Annual Conference and Exposition: Staying in Tune with Engineering Education, pp. 821-828, June 2003.

[11] K. K. Tan, T. H. Lee, and F. M. Leu, "Development of a Distant Laboratory using LabVIEW," International Journal of Engineering Education, vol. 16, no. 3, pp. 273-282, 2000.
[12] A. K. M. Azad, A. Otieno, O. Ghrayeb, and N. Anand, "Internet based experiments for physical laboratory set-up," in Proceedings of the ASEE Annual Conference and Exposition: Staying in Tune with Engineering Education, pp. 3337-3347, June 2003.

[13] I. Gustavsson, J. Zackrisson, H. Akesson, L. Hakansson, I. Claesson, and T. Lago, "Remote operation and control of traditional laboratory equipment," International Journal of Online Engineering, vol. 2, no. 1, 2006.

[14] Y. Zhou, J. Jian-Jun, and F. Shao-Chun, "A LabVIEWbased, interactive virtual laboratory for electronic engineering education," International Journal of Engineering Education, vol. 21, no. 1, pp. 94-102, 2005.

[15] D. Z. Deniz, A. Bulancak, and G. Özcan, "A novel approach to remote laboratories," in Proceedings of the 33rd ASEE/IEEE Frontiers in Education Conference, pp. T3E-8-T3E-12, Boulder, Colo, USA, November 2003.

[16] W. H. Blanton, "Distance learning opportunities for electronic engineering technology graduates of community colleges," in Proceedings of the ASEE Annual Conference and Exposition, pp. 3933-3940, June 2004.

[17] C. Sapijaszko and G. I. Sapijaszko, "An innovative electronics laboratory system for on campus and distance learning applications," in Proceedings of the ASEE Annual Conference and Exposition, pp. 7371-7392, June 2004.

[18] I. Gustavsson, "User-defined electrical experiments in a remote laboratory," in Proceedings of the ASEE Annual Conference and Exposition: Staying in Tune with Engineering Education, pp. 4799-4808, June 2003.

[19] I. Gustavsson, "A remote access laboratory for electrical circuit experiments," International Journal of Engineering Education, vol. 19, no. 3, pp. 409-419, 2003.

[20] M. Naghedolfeizi, S. Arora, and S. Garcia, "Survey of Lab VIEW technologies for building Web/Internet-enabled experimental setups," in Proceedings of the ASEE Annual Conference and Exposition: Vive L'ingenieur, pp. 7897-7906, June 2002.

[21] A. Mani and C. Patvardhan, "A remote experimental setup for performing experiments on temperature sensors," in Proceedings of the International Conference on Engineering Education (ICEE '06), San Juan, Puerto Rico, usa, 2006.

[22] "Elettronica Veneta's Acceleration Transducers Module's Manual".

[23] A. Mani and C. Patvarchan, "A low cost programmable power supply," Journal of Instrument Society of India, vol. 36, no. 3, pp. 212-219, 2006. 


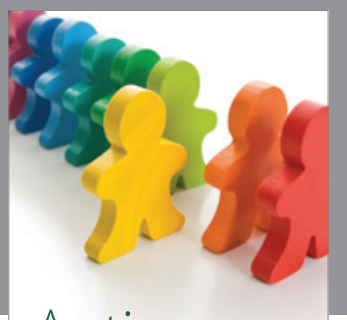

Autism

Research and Treatment
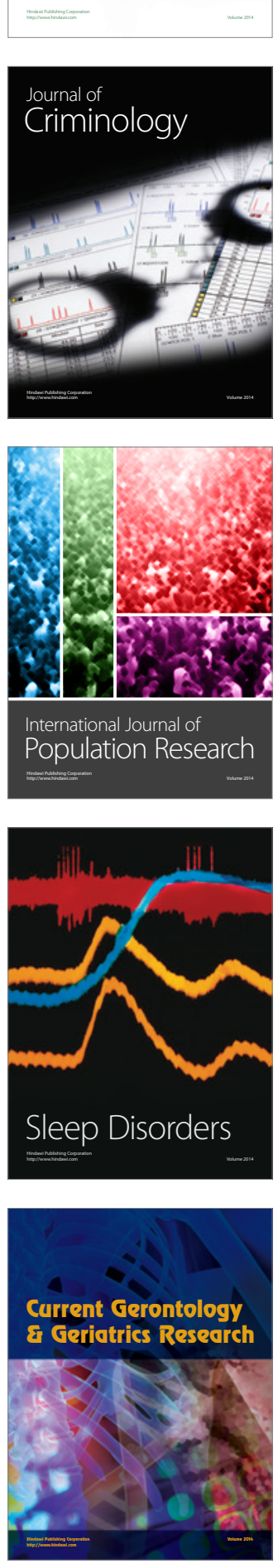
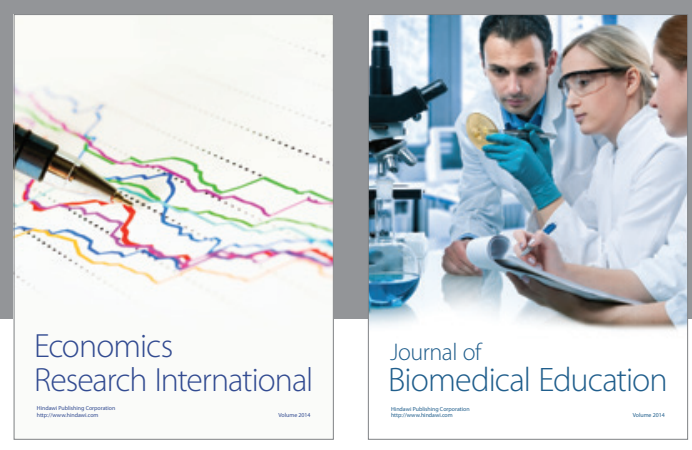

Journal of

Biomedical Education

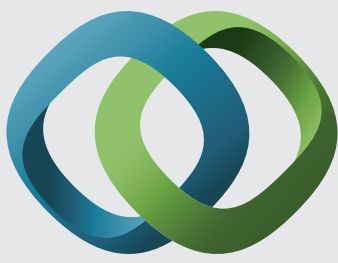

\section{Hindawi}

Submit your manuscripts at

http://www.hindawi.com
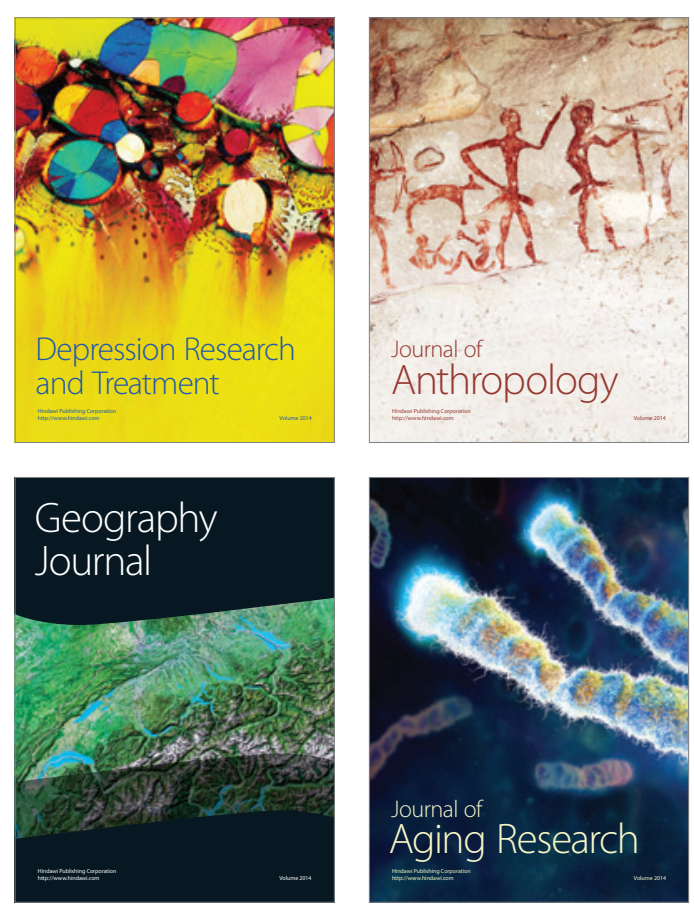

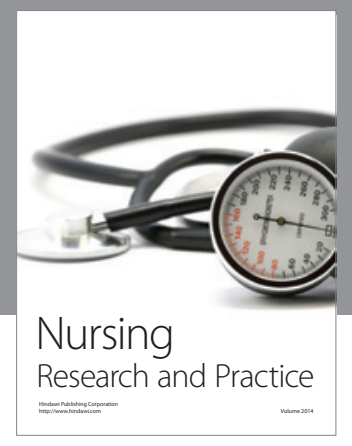

Nursing

Research and Practice

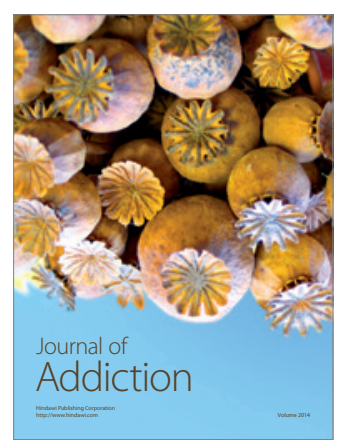

Child Development

Research

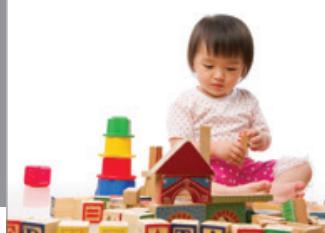

迥
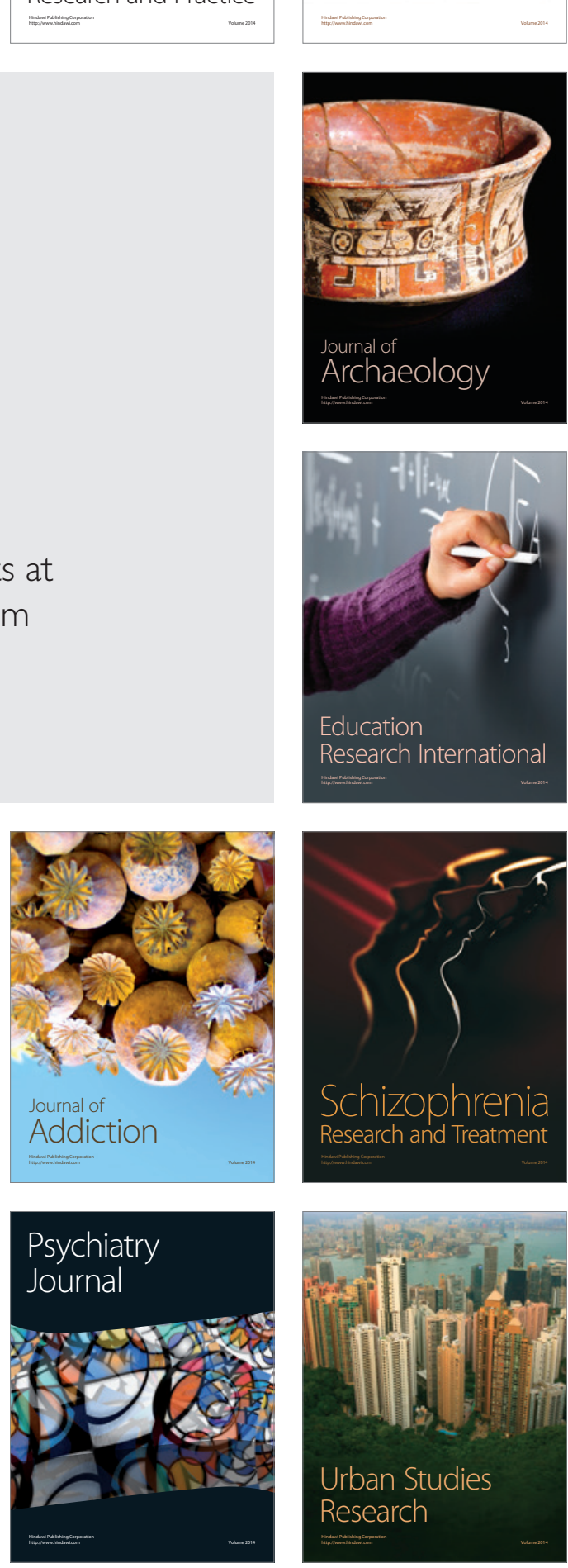